\title{
Capital-labour-energy substitution in a nested CES framework: A replication and update of Kemfert (1998)
}

Henningsen, Arne; Henningsen, Geraldine; van der Werf, Edwin

Published in:

Energy Economics

Link to article, DOI:

10.1016/j.eneco.2017.12.019

Publication date:

2019

Document Version

Peer reviewed version

Link back to DTU Orbit

Citation (APA):

Henningsen, A., Henningsen, G., \& van der Werf, E. (2019). Capital-labour-energy substitution in a nested CES framework: A replication and update of Kemfert (1998). Energy Economics, 82, 16-25.

https://doi.org/10.1016/j.eneco.2017.12.019

\section{General rights}

Copyright and moral rights for the publications made accessible in the public portal are retained by the authors and/or other copyright owners and it is a condition of accessing publications that users recognise and abide by the legal requirements associated with these rights.

- Users may download and print one copy of any publication from the public portal for the purpose of private study or research.

- You may not further distribute the material or use it for any profit-making activity or commercial gain

- You may freely distribute the URL identifying the publication in the public portal 


\section{Accepted Manuscript}

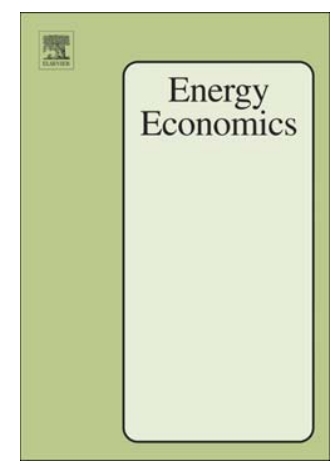

Capital-labour-energy substitution in a nested CES framework: a replication and update of Kemfert (1998)

Arne Henningsen, Geraldine Henningsen, Edwin van der Werf

PII:

DOI:

S0140-9883(17)30439-5

Reference: $\quad$ ENEECO 3858

To appear in: $\quad$ Energy Economics

Received date: 1 August 2017

Revised date: 29 November 2017

Accepted date: 14 December 2017

Please cite this article as: Henningsen, Arne, Henningsen, Geraldine, Werf, Edwin van der, Capital-labour-energy substitution in a nested CES framework: a replication and update of Kemfert (1998), Energy Economics (2018), doi:10.1016/j.eneco.2017.12.019

This is a PDF file of an unedited manuscript that has been accepted for publication. As a service to our customers we are providing this early version of the manuscript. The manuscript will undergo copyediting, typesetting, and review of the resulting proof before it is published in its final form. Please note that during the production process errors may be discovered which could affect the content, and all legal disclaimers that apply to the journal pertain. 


\title{
Capital-labour-energy substitution in a nested CES framework: a replication and update of Kemfert (1998)
}

\author{
Arne Henningsen ${ }^{\mathrm{a}}$, Geraldine Henningsen ${ }^{\mathrm{b}, *}$, Edwin van der Werf $\mathrm{f}^{\mathrm{c}, \mathrm{d}}$ \\ ${ }^{a}$ Department of Food and Resource Economics, University of Copenhagen, \\ Rolighedsvej 25, 1958 Frederiksberg C, Denmark \\ ${ }^{b}$ Department of Management Engineering, Technical University of Denmark, \\ Produktionstorvet 426, 2800 Kgs. Lyngby, Denmark \\ ${ }^{c}$ Environmental Economics and Natural Resources Group, Wageningen University E Research, \\ PO Box 8130, 6700EW Wageningen, The Netherlands \\ ${ }^{d}$ CESIfo GmbH, Poschingerstr. 5, 81679 Munich, Germany
}

\begin{abstract}
The ease with which firms can substitute away from energy to other inputs is an important determining factor in the costs of climate change mitigation policies. Climate policy simulation models usually represent this substitutability by using the Constant Elasticity of Substitution (CES) function with parameter values often taken from econometric studies. Hence, the accuracy of the estimated substitution parameters has a strong influence on the validity of the climate policy simulation. In this article, we attempt to replicate the results presented in a widely cited article in this field: Kemfert (1998) ('Estimated substitution elasticities of a nested CES production function approach for Germany', Energy Economics, 20, 249-264). We first use the data and software reported in that article and compare our results with those reported in the original study. We then test the same data and a new, more recent, data set on German industrial data with an improved econometric approach. Despite applying various approaches and modifications, we are not able to replicate the results in Kemfert (1998). We furthermore conclude that the data sets that are typically used to estimate nested CES functions often have too few observations and too little independent variation of the explanatory variables to obtain reliable estimates when using a direct non-linear approach. ${ }^{1}$
\end{abstract}

JEL classification: Q41, Q43, C50

Keywords: CES, replication study, elasticity of substitution, energy, climate policy

\footnotetext{
${ }^{1}$ Abbreviations: CES $=$ Constant elasticity of substitution; IAM = Integrated assessment model; $\mathrm{CGE}=$ Computable general equilibrium; RSS = Residual sum of squares; KLE = Capital, labour, energy

* Corresponding author. Senior authorship is equally shared by all three authors.

Email addresses: arne@ifro.ku.dk (Arne Henningsen), gehe@dtu.dk (Geraldine Henningsen),

Preprint submitted to Energy Economics

February 12, 2018
} 


\section{Introduction}

Climate change mitigation policies are for the most part aimed at decreasing the use of carbon-based energy sources. The costs of such policies are therefore to a large extent determined by the ease with which firms can move away from fossil fuels, and from energy to other inputs. The ease with which firms can switch between inputs is determined by the elasticity of substitution. Hence, the values of the elasticities of substitution between capital, labour and energy are key in determining the costs of simulated climate policy scenarios (Jacoby et al., 2006). To simulate climate policy scenarios, most integrated assessment models (IAMs) and computable general equilibrium (CGE) models use production functions of the constant elasticity of substitution (CES) type in which multiple inputs are combined in a nested structure - see for example the models WITCH (Carrara and Marangoni, 2017) and REMIND (Bauer et al., 2012). The values of the substitution elasticities used in IAMs and CGE models are usually picked from econometric studies that estimate elasticities of substitution, often using CES production functions. Hence, the quality of the estimates of the substitution elasticities, is crucial to ensure valid policy simulation.

In this article we try to replicate and update the results from one of these econometric studies: Kemfert (1998). Kemfert (1998) is an often-cited article that presents the results of estimations of CES production functions with capital, labour and energy as inputs, using data for West-Germany. ${ }^{2}$ Several policy simulation studies refer to this article for one or more of the values of the elasticity of substitution used in the simulation model (e.g., Wissema and Dellink, 2007; Dong and Whalley, 2011; Turner and Hanley, 2011; Meng, 2014). Kemfert (1998) reports estimated elasticities for the German industry for 1960-1993 and for seven industrial sub-sectors for 1970-1988. It was one of the first studies to estimate nested CES functions directly, using a non-linear estimation technique, rather than estimating linear models based on profit maximisation or cost minimisation. One of the main objectives of the paper was to test which nesting structure fits the data best, i.e., whether capital should be first combined with labour using one CES function, the result of which is combined with energy using another CES function (a (KL)E nesting structure), or that capital should first be combined with energy (a (KE)L nesting structure), or labour and energy should be combined first (a (LE)K nesting structure).

The objectives of our article are (1) to have a close look at the data and methods described in Kemfert (1998), (2) to see whether we can replicate the results reported in that study with the original data and method (using various algorithms and starting

edwin.vanderwerf@wur.nl (Edwin van der Werf)

${ }^{2}$ Until October 22, 2017, Kemfert (1998) had 64 citations according to Scopus and 232 citations according to GoogleScholar. 
values for estimating non-linear functions in SHAZAM), (3) to see whether we can replicate the results reported in Kemfert (1998) using up-to-date methods, and (4) to update results using a more recent data set and up-to-date methods.

We find problems with the dependent variable used in Kemfert (1998) and with the reported data for the 'capital' input. Nevertheless, we use the data reported in Kemfert (1998) to replicate the analysis with the software that was used in the original study; in addition we apply an improved optimization algorithm (using the add-on package micEconCES for R; Henningsen and Henningsen, 2011b) to estimate nested CES functions (Henningsen and Henningsen, 2012). Furthermore, in order to find valid replication results, we estimate various model specifications, apply multiple optimisation algorithms, and use various methods to derive valid starting values for the optimization algorithm. Unfortunately, although our results derived with the two statistical software packages are reasonably consistent, only very few of our estimated substitution elasticities are close to the values reported in Kemfert (1998).

Given that the data set used in Kemfert (1998) has serious problems and is rather old to analyse current policy issues (as it only refers to the former German Federal Republic, it is no longer valid for today's Germany), we furthermore construct a new and corrected data set covering similar sectors as those in Kemfert (1998) for the years 1991-2014 and the entire Federal Republic of Germany. Applying this new and more recent data set, we estimate the CES production function with capital, labour, and energy as inputs for the three nesting structures - $(K L) E,(K E) L$, and $(L E) K$ - using the above-mentioned improved optimisation algorithm for nested CES functions. Even for this data set we find elasticities of substitution ranging from close to zero to infinity, with large standard errors. We conclude that the data sets that are typically used to estimate nested CES functions often have too few observations and too little independent variation of the explanatory variables to obtain reliable estimates when using a direct non-linear approach.

The remainder of this article is organised as follows. In section 2, we present the model to be estimated and the estimation method used in Kemfert (1998). In section 3, we present our replication study: we analyse the data used in Kemfert (1998) and present the results of various attempts to replicate the results from that article as well as compare our results to the results reported in Kemfert (1998). In section 4, we present the new data set and the results of our estimations based on this more recent data set. We conclude in section 5 . 


\section{Models and method}

Following the notation for parameters as used in Kemfert (1998), the three-input nested CES production function can be written as follows:

$$
Q_{t}=e^{m t} A\left[a\left(b K_{t}^{-\alpha}+(1-b) E_{t}^{-\alpha}\right)^{\frac{\beta}{\alpha}}+(1-a) L_{t}^{-\beta}\right]^{-\frac{1}{\beta}} .
$$

In this particular design of the three-input nested CES function, capital $K$ and energy $E$ are first combined into an intermediate input using a two-input CES function with share parameter $b$ and substitution parameter $\alpha$. This intermediate input is subsequently combined with labour $L$ into output $Q$, using another CES production function with share parameter $a$ and substitution parameter $\beta$. The constant elasticities of substitution can be derived using

$$
\sigma_{i n}=\frac{1}{1+\alpha}
$$

and

$$
\sigma_{\text {out }}=\frac{1}{1+\beta}
$$

where (2) presents the calculation of the elasticity of substitution for the inner nest (i.e., between capital and energy in case of the nesting structure in equation (1)) and (3) presents how the elasticity of substitution between the composite from the inner nest and the third input is calculated. Furthermore, $A$ denotes a general productivity parameter, whilst $m$ denotes a constant (Hicks-neutral) annual rate of technological change and $t$ denotes time in years. The variable on the left-hand side, $Q$, denotes the output that results from combining capital, labour, and energy. In IAMs and CGE models, this output can be further combined with intermediate inputs to produce a final output. As it is common in such models, equation (1) exhibits constant returns to scale. It is important to note that the nesting structure presented in (1) is only one possible structure: it combines capital and energy first and can therefore be denoted by $(K E) L$; alternative nesting structures are $(K L) E$ and $(E L) K$.

The substitution parameters can be estimated using one of two different approaches. With the 'economic approach' the elasticity of substitution is estimated from equations based on the first-order conditions of a profit-maximisation or cost-minimisation problem (see e.g., Prywes, 1986; Chang, 1994; Van der Werf, 2008). However, this approach requires price data for all inputs in all sectors, which might not always be available. Furthermore, this approach assumes the absence of market distortions, which is not always the case - in particular for energy markets (Klump et al., 2011). Alternatively one can use a 'technical approach' where equation (1) is directly estimated using quantity 
data only (see e.g., Koesler and Schymura, 2015; Heun et al., 2017). This is the approach used in Kemfert (1998).

A direct estimation of (1) requires a non-linear estimation method. Kemfert (1998) reports that this equation and the equations for the alternative nesting structures have been estimated using the econometrics software package SHAZAM. Unfortunately, whilst acknowledging the non-linearity of the equation in (1), the article does not provide further details about the exact methodology used.

\section{Replication of Kemfert (1998)}

Appendix A in Kemfert (1998) reports the data used in the analysis of that article. In the next subsection we have a closer look at these data. We subsequently present the estimation methods used in our replication, after which we present the results of our replication of Kemfert (1998), based on the data reported in that article.

\subsection{Data}

Kemfert (1998) reports the data and estimated substitution elasticities for eight sectors: German Industry (which covers "the German industry as a whole"; Kemfert, 1998, p.253) and the sub-sectors Chemical industry, Stone and earth, Non-ferrous, Iron, Vehicle, Paper, and Food. Data reportedly come from the German Statistical Office (DESTATIS) but unfortunately the exact data sources used are not reported in the article. Furthermore, the article omits to mention the sector classification used. The time period covered is 1960-1993 for the first sector and 1970-1988 for all sub-sectors, whilst the years 1973-1975 are excluded because of the economic disruptions from the oil crisis. 3

According to the data reported in Appendix A of Kemfert (1998), the dependent variable in all regressions is "gross value added at prices of 1991" (Kemfert, 1998, p.263 and p.264). Data for $K$ denote "gross fixed capital stock at prices of 1991" (p.263) for German Industry and "Gross fixed capital formation" (p.265) for the sub-sectors. Data for $L$ denote persons employed. Energy consumption $E$ is reported in GWh for German Industry and in "Mio t SKE (GWh)" for the other sectors (p.261), where "Mio t SKE" probably means million tons of coal equivalents (Steinkohle Equivalent). Since the conversion factor between million tons of coal equivalents and GWh is of multiple orders of magnitude, the numbers in the table in Appendix A of Kemfert (1998) suggest that data for energy consumption are in GWh.

\footnotetext{
${ }^{3}$ For the sub-sectors, data on value added, labour, and capital are reported for 1970-1992, but since data for energy are only reported for 1988 the regression analyses for these sectors cannot go beyond that year.
} 


\subsubsection{Dependent variable}

As noted above, the dependent variable in the regressions in Kemfert (1998) is gross value added. It is, however, important to note that the explanatory variables do not only include capital and labour, but also energy. Hence the production function maps these inputs not just onto value added, but onto the sum of value added and energy costs (Van der Werf, 2008; Brockway et al., 2017). Using value added as dependent variable, instead of the sum of value added and the value of energy, will lead to unreliable estimates of the substitution parameters due to an incorrectly calculated dependent variable, which is even correlated with an explanatory variable, i.e., energy use. A first conclusion from inspecting the data reported in Kemfert (1998) is therefore that the elasticities reported in that article may suffer from biased estimates.

\subsubsection{Capital data}

Next, we have a close look at the data for the capital stock, as reported in Kemfert (1998). When plotting the capital series for the sector German Industry one can observe three jumps in the values: in 1970, 1980 and 1989. This could, for example, come from a change in the definition of 'gross fixed capital stock' used by the source of the data. The data for the other sectors show a different pattern: no clearly distinctive jumps but highly volatile series. Indeed, $15 \%$ of the observations show year-on-year changes larger than $25 \%$, which can be considered to be very large for a capital stock. Furthermore, it shows one obvious outlier in the data: there is a $300 \%$ increase in the capital stock of the sector Vehicle in the year 1983, which is followed by a $76 \%$ decrease that brings the level of the capital stock in line with observations for other years.

To further assess the capital data, we plotted ratios between capital and value added $(K / Y$ ratios $)$ see figures A.1 and A.2 in Section A of the On-line Appendix. The $K / Y$ ratio for German Industry indeed shows three jumps. Furthermore, the ratios are constant in the period up to each jump: for 1960-1969 K/Y=0.0535, for 1970-1979 $K / Y=0.0743$ and for $1980-1988 K / Y=0.0983$. After 1988 , the ratio is no longer a constant. The $K / Y$ ratios for the sub-sectors, on the other hand, are highly volatile for the entire period (see Figure A.2), reflecting the high volatility of the capital stock data for these sectors mentioned above.

Figures A.1 and A.2 also show that the $K / Y$ ratio for the German Industry is always smaller than 0.15 , whilst for the other sectors it is always smaller than 0.8 and for most years even smaller than 0.4. To put these numbers in perspective, we created a data set covering comparable sectors and the same period, using data from the German Federal Statistical Office (see Section A.2 of the On-line Appendix for the construction of this data set), and compare the capital-value added ratios from this data set against the ratios

reported in Kemfert (1998). We were able to construct $K / Y$ ratios for five sectors that 
are in name and employment numbers similar to the sectors in Kemfert (1998), including German Industry. Figure A.3 shows that the levels of the $K / Y$ ratios range from 1.7 to 6.4 and that they are much less volatile than the $K / Y$ ratios resulting from the data reported in Kemfert (1998). Next, we created $K / Y$-ratios for similar sectors using data from the World Input-Output Database (WIOD) (Timmer et al., 2012) - the data used in Koesler and Schymura (2015). Although these data are only available from 1995 onwards, we find $K / Y$-ratios ranging from 1.4 to 4.1 and a volatility that is comparable to that showed in Figure A.3. Finally, we calculated $K / Y$-ratios for the data used in Van der Werf (2008) and find values from 1.5 to 3.1 and a volatility that is comparable to that found in the new data from the Federal German Statistical Office and in the data used by Koesler and Schymura (2015). These observations suggest that the capital data reported in Kemfert (1998) cannot be considered to be representative capital series.

The appendix to Kemfert (1998) reports that the capital data for the sub-sectors (but not the data for German Industry) are data for "Gross fixed capital formation". It can be shown mathematically that, if gross fixed capital formation approximately equals gross fixed capital stock up to a constant scaling factor, then data on the former can be used to estimate the elasticities of substitution between capital and other inputs. Using the data described in Section A.2 of the On-line Appendix, we constructed ratios for gross fixed capital formation and gross fixed capital stock for the period 1970-1991. Within each sector, the maximum value for this ratio is at least twice as large as the minimum value (see Figure A.4), which indicates substantial variation in the investmentstock ratio. Based on these observations we conclude that gross fixed capital investment is not (approximately) a constant fraction of gross fixed capital formation. Hence, the capital series for the sub-sectors, as reported in Kemfert (1998), are suboptimal data to estimate the elasticity of substitution between the stock of capital and other inputs. ${ }^{4}$

These observations show that there are problems with the variability and the levels of the data for the capital series reported in Kemfert (1998) for all sectors. A second conclusion from inspecting the data reported in Kemfert (1998) is therefore that using the capital data reported in that paper as data for the stock of capital in estimates of CES production functions may lead to unreliable estimates of the elasticities of substitution between the stock of capital and other inputs.

\subsubsection{Outliers}

Having had a close look at the capital series reported in Kemfert (1998), we now have a look at the data for the other variables. When plotting the data for value added,

\footnotetext{
${ }^{4}$ Note that policy simulation models use the stock of capital, rather than capital formation or investment, as input in their production function.
} 
there is one obvious outlier: a $707 \%$ increase for Chemical industry in 1977, followed by a 90\% decrease in 1978 that brings the level back in line with values for other years. Although there are other large jumps in the data, it is less obvious whether these are true outliers. Value added in the Paper sector increases by $104 \%$ in 1981, but is followed by another large increase (51\%) in the year thereafter, which in turn is followed by a $32 \%$ decrease in 1983 and a $46 \%$ decrease in 1984. In the employment data we observe a $47 \%$ increase for Chemical industry in 1983, followed by a $33 \%$ decrease in 1984 . It is unclear to which degree these outliers affect the estimation in Kemfert (1998) or whether they result from typing errors in the processing of the tables in the appendix of Kemfert (1998). Furthermore, there are a few large jumps in the data in the period 1973-1975 (a 111\% increase in value added for Non-ferrous in 1974; and a $118 \%$ increase in employment in the Vehicle sector in 1975), but these years are omitted in the econometric analysis in Kemfert (1998).

To summarise, there are serious problems with the data reported in Appendix A of Kemfert (1998) that may lead to unreliable estimates of the elasticities of substitution. In the remainder of this section we ignore most of these problems and attempt to replicate the results reported in Kemfert (1998) with the data reported in that article. We only correct the two obvious outliers described above as these are most likely the results of typing errors. ${ }^{5}$ In Section 4 we present results of estimations based on an improved data set.

\subsection{Estimation method}

As noted above, Kemfert (1998) does not contain details about the estimation method, other than that a non-linear regression method was used in the statistical software SHAZAM. We tried to replicate her results using the same software and method. However, as SHAZAM only offers a limited range of options for non-linear regressions which are required to estimate the CES function in a direct approach as done by Kemfert, we also tried to replicate Kemfert's results using R (R Core Team, 2017). In the remainder of this subsection we give an overview of the methods and specifications used for our replications in SHAZAM and $R$.

\subsubsection{Additive and multiplicative error terms}

Equation (1) can be estimated by adding an error term $\epsilon$ to the right-hand side of this equation, like implied in Kemfert (1998). An alternative specification, suggested, e.g., by Judge et al. (1982, p. 652) and White (1992, p.372), multiplies the right-hand side of

\footnotetext{
${ }^{5}$ The value added for the Chemical sector in the year 1977 has been changed from 654,784 to 65,474 . The capital stock for the Vehicle sector in the year 1983 has been changed from 85,598 to 25,598.
} 
equation (1) by the exponential function of an error term $\left(e^{\epsilon}\right)$ and takes logarithms of both sides of this equation, which gives:

$$
\ln \left(Q_{t}\right)=m t+\ln (A)-\frac{1}{\beta} \ln \left[a\left(b K_{t}^{-\alpha}+(1-b) E_{t}^{-\alpha}\right)^{\frac{\beta}{\alpha}}+(1-a) L_{t}^{-\beta}\right]+\epsilon
$$

As a result, one estimates a comparable model as in equation (1) but with a multiplicative rather than an additive error term. Whilst an additive error term measures the deviations between the observed and 'fitted' output quantities in absolute terms, a multiplicative error term measures these deviations in relative terms. In most empirical applications, a multiplicative error term leads to more homoskedastic residuals than an additive error term. In our replications, we estimate the CES function both with an additive error term (1) and with a multiplicative error term (4).

\subsubsection{Mean-scaled and non-mean scaled data}

As the tables in Appendix A of Kemfert (1998) indicate, many values in the data set are very large in absolute numbers, with maximum values of over 300,000 for value added (in million DM) and over 30,000 for capital (in million DM) and energy (in GWh). As a result, the values for $K^{-\alpha}, E^{-\alpha}$, or $L^{-\beta}$ in equations (1) and (4) can get close to zero, which causes numerical inaccuracies. These numerical inaccuracies likely result in inaccurate calculations of the 'fitted' output quantities. Thus, affecting the sum of squared residuals (see equations (5) and (6)), which is the objective function to be minimized in the estimations. Particularly in non-linear estimations, these numerical inaccuracies can strongly decrease the accuracy of the estimates.

It can be shown, however, that the substitution parameters ( $\alpha$ and $\beta$ ) and the parameter for technical progress $(m)$ of a nested CES function, as well as the $R^{2}$ of the resulting regression are invariant to units of measurement, i.e., these values are not affected by multiplying or dividing all values of a variable by the same number (e.g., dividing the value added by 1,000 converts its units of measurement from million DM to billion DM). ${ }^{6}$ Hence, mean-scaling the input quantities so that they have values around one reduces numerical inaccuracies and still allows for comparability with the results reported in Kemfert (1998). 7 In our replication study, we estimate the models both with the original data and with the mean-scaled data.

\footnotetext{
${ }^{6}$ The share parameters, $a$ and $b$, and the general productivity parameter $A$ are affected by changing the units of measurement. However, these parameters are not reported in Kemfert (1998) and are not coefficients of interest in this replication study. Furthermore, the partial production elasticities of the inputs, which give more easily interpretable measures of the contributions of the individual inputs than the share parameters, are unaffected to changes in the units of measurement.

7 Klump and de La Grandville (2000) and Klump et al. (2011) recommend normalising the data, i.e., dividing all values of a variable by its value in a 'base' period. This has a very similar effect as meanscaling the data, i.e., dividing all values of a variable by its mean value. While Klump et al. (2011)
} 


\subsubsection{Optimisation algorithms and starting values}

To estimate the parameters of the nested CES function in (1) using nonlinear least squares, one has to find the parameters $A, m, a, b, \alpha$, and $\beta$ that minimize the sum of squared residuals:

$$
\min _{A, m, a, b, \alpha, \beta} \sum_{t}\left(Q_{t}-e^{m t} A\left[a\left(b K_{t}^{-\alpha}+(1-b) E_{t}^{-\alpha}\right)^{\frac{\beta}{\alpha}}+(1-a) L_{t}^{-\beta}\right]^{-\frac{1}{\beta}}\right)^{2}
$$

or

$\min _{A, m, a, b, \alpha, \beta} \sum_{t}\left(\ln \left(Q_{t}\right)-m t-\ln (A)+\frac{1}{\beta} \ln \left[a\left(b K_{t}^{-\alpha}+(1-b) E_{t}^{-\alpha}\right)^{\frac{\beta}{\alpha}}+(1-a) L_{t}^{-\beta}\right]\right)^{2}$,

for additive or multiplicative error terms, respectively. Since these objective functions are non-linear in the parameters that are to be estimated, estimation of the parameters requires the use of a non-linear optimisation algorithm.

It is not stated in Kemfert (1998), which optimisation algorithm in SHAZAM was used for obtaining the reported parameter estimates. Therefore, we use in our replication study all three optimisation algorithms that were available in SHAZAM in 1998: Davidon-Fletcher-Powell (DFP, the default in SHAZAM), Broyden-Fletcher-GoldfarbShanno (BFGS), and a modification of DFP (DFP 2). All three algorithms are of the Quasi-Newton type, which is a solver class for non-linear optimisation that is often used for non-linear regression. Although some Quasi-Newton type algorithms, like the BFGS algorithm, prove robust in many practical applications, these types of optimisation algorithms often have problems in estimating CES functions, because the objective functions for estimating CES functions (i.e., (5) or (6) in our case) usually have very irregular surfaces with many local minima (Henningsen and Henningsen, 2012). When objective functions have these characteristics, Quasi-Newton type optimisation algorithms do not necessarily find the global minimum of the objective function but often converge to a local minimum that is close to the starting values (Kelley, 1999). Hence, the estimates of the parameters of the CES function often depend on the starting values set by the analyst. Circumventing this problem requires the use of starting values that are already close to the global minimum of the objective function (Henningsen and Henningsen, 2012).

As the starting values used by Kemfert (1998) are unknown to us, we set them within the most reasonable ranges. We set the starting value for the technical progress parameter to $m=0.02$; the share parameters, $a$ and $b$, are set so that the partial

argue that normalizing the data makes it easier to find appropriate starting values and to interpret the (estimated) parameters, we point out that improved numerical accuracy is an additional advantage of mean-scaling or normalising the data. 
production elasticities of energy, capital, and labour are $0.1,0.2$, and 0.7 , respectively. ${ }^{8}$ The general productivity parameter, $A$, is set so that the predicted output quantity for the mean values of the input quantities equals the mean observed output quantity (using the previously defined starting values for $m, a$, and $b$, and the starting values for $\alpha$ and $\beta$ described below). However, given the above discussion on the convergence towards a global minimum, one needs to bear in mind, that these starting values are only qualified guesses and not necessarily optimal. Therefore, in order to increase our chances to find the global minimum of the objective function, we estimate the models with different sets of starting values, where we vary the starting values for the two substitution parameters, $\alpha$ and $\beta$, over the set $\alpha=\beta \in\{-0.5,0.01,0.5,0.9\}$. Out of the estimations with the different sets of starting values, we choose those estimates that result in the smallest sum of squared residuals (SSR) in equation (5) or (6), respectively.

In addition to SHAZAM, we conduct the replication analysis in the statistical software R (R Core Team, 2017) using the add-on package micEconCES (Henningsen and Henningsen, 2011a,b), which can address several problems that are inherent in estimations of the CES function (Henningsen and Henningsen, 2012): (i) finding the global minimum of the objective function and (ii) producing parameter estimates which are in line with economic theory $(0<a, b<1 ; \alpha, \beta \geq-1)$.

In order to address these two points, Henningsen and Henningsen (2011a) compare the performance of several different optimisation algorithms suitable for estimating the non-linear CES function. The optimal choice to address point (i) is the LevenbergMarquardt (LM) algorithm (Marquardt, 1963) which generally was more successful in minimizing the sum of squared residuals (SSR) than other algorithms. The optimal choice to address point (ii) is the PORT algorithm (Gay, 1990), a Quasi-Newton type algorithm, which performed best among all algorithms that can impose parameter restrictions. Following these findings, we will restrict the further discussion on these two algorithms, where the PORT algorithm will be used to impose parameter restrictions such that the results are in line with economic theory. As neither the LM nor the PORT algorithm are global optimisation algorithms, and hence, are prone to the same problem as discussed above, we combine both optimisation algorithms with a grid search over the substitution parameters $\alpha$ and $\beta$, i.e., we estimate the remaining parameters of the CES function by pre-imposing fixed parameter values for the substitution parameters.

In order to increase the probability that we find the global minimum of the objective function, and, hence, the least-squares estimates of the parameters of the CES function,

\footnotetext{
${ }^{8}$ In empirical applications on production data, the partial production elasticities for capital and labour are often found to be in the ranges of $0.75-0.8$ and $0.2-0.25$, respectively. We furthermore assume that the partial production elasticity of energy is considerably smaller than for capital.
} 
we estimate the nested CES function with three different approaches for each of the two optimisation algorithms (LM and PORT):

1. Estimating the nested CES function using the default starting values used by the micEconCES package (i.e., $m=0.015, a=b=0.5, \alpha=\beta=0.25$, and $A$ as described above (for estimations in SHAZAM).

2. Running a two-dimensional grid search with 61 different values between -1 and 14 for each of $\alpha$ and $\beta$, i.e., for elasticities of substitution between 0.067 and infinity. For each of the $61^{2}=3721$ estimations, the values of $\alpha$ and $\beta$ are fixed at values at the respective grid point, whilst the other parameters $(m, A, a, b)$ are estimated using the same starting values as used in step 1. Out of these 3721 estimations, the estimation that results in the smallest sum of squared residuals is chosen.

3. Estimating the nested CES function using the parameters obtained in step 2 as starting values.

For each of the two optimisation routines (LM and PORT), we choose the approach (1-3) that results in the smallest sum of squared residuals. By following this procedure, we increase the likelihood of finding the set of parameters that results in a global minimum of the objective function for each of the specifications outlined in the previous sub-sections. In the estimations with the PORT algorithm, we restrict the values of the parameters so that they are in line with economic theory (e.g., fulfillment of the monotonicity condition for the share parameters, i.e. $0<a, b<1$, and convex isoquants for the substitution parameters, i.e., $\alpha, \beta \geq-1$ ).

\subsection{Results and discussion}

In total, we ran 144 different regressions for each sector in SHAZAM ${ }^{9}$ and 36 different regressions for each sector in $\mathrm{R}^{10}$. Table 1 summarises the different estimation approaches used in SHAZAM and R:

Table 2 presents an excerpt of the results from the analyses in SHAZAM and R for the sector German Industry (results for the sub-sectors are available in Section B of the On-line Appendix). For each nesting structure and error term specification, we report the estimates of our regressions with SHAZAM, LM, and PORT that give the smallest sums of squared residuals and we compare them with the corresponding estimates of Kemfert (1998). As estimation results can always slightly deviate because of numerical inaccuracies, different computer systems, and different versions of the statistical software, and as

\footnotetext{
${ }^{9} 3$ different nesting structures $\times 2$ different specifications of the error term $\times 2$ different scales of the variables (mean-scaling and non-mean-scaling) $\times 3$ different optimisation algorithms $\times 4$ different sets of starting values.

${ }^{10} 3$ different nesting structures $\times 2$ different specifications of the error term $\times 2$ different optimisation algorithms $\times 3$ different approaches.
} 
Table 1: Estimation specifications for the replications in SHAZAM and R

\begin{tabular}{|c|c|c|c|c|c|}
\hline Software & Nesting & Error term & Scaling & Algorithms & Starting values \\
\hline SHAZAM & $\begin{array}{l}\text { All } 3 \text { nesting } \\
\text { structures } \\
\text { are included }\end{array}$ & $\begin{array}{l}\text { Additive } \\
\text { and mul- } \\
\text { tiplicative } \\
\text { error terms }\end{array}$ & $\begin{array}{l}\text { Mean scaled } \\
\text { and non- } \\
\text { mean scaled } \\
\text { variables }\end{array}$ & $\begin{array}{l}\text { DFP; } \\
\text { BFGS; } \\
\text { DFP 2 }\end{array}$ & $\begin{array}{l}\text { Fixed starting val- } \\
\text { ues for } A, a, b \text {, } \\
\text { and } m \text { based on eco- } \\
\text { nomic considerations } \\
\text { and varying start- } \\
\text { ing values for the } \\
\text { substitution param- } \\
\text { eters } \alpha=\beta \in \\
\{-0.5,0.01,0.5,0.9\}\end{array}$ \\
\hline $\mathrm{R}$ & $\begin{array}{l}\text { All } 3 \text { nesting } \\
\text { structures } \\
\text { are included }\end{array}$ & $\begin{array}{l}\text { Additive } \\
\text { and mul- } \\
\text { tiplicative } \\
\text { error terms }\end{array}$ & $\begin{array}{l}\text { Only mean } \\
\text { scaled vari- } \\
\text { ables }\end{array}$ & $\begin{array}{l}\text { LM; PORT } \\
\text { with param- } \\
\text { eter restric- } \\
\text { tions }\end{array}$ & $\begin{array}{l}\text { Default } \\
\text { ing values } \\
\text { micEconCES; } \\
\text { dimensional } \\
\text { search over } \alpha \text { grid } \\
\beta ; \quad \text { starting values } \\
\text { from best performing } \\
\text { regression in the grid } \\
\text { search }\end{array}$ \\
\hline
\end{tabular}

we do not know the exact specifications and the starting values underlying the results in Kemfert (1998), we include a range of tolerance into the comparison of our results to those of Kemfert (1998). Our estimates are considered to be 'close' to those of Kemfert (1998) if the technical progress parameter, $m$, is within the range of \pm 0.01 or $\pm 10 \%$ and the substitution parameters, $\alpha$ and $\beta$, and the corresponding elasticities of substitution, $\sigma_{\text {in }}$ and $\sigma_{\text {out }}$, are within the range of \pm 0.025 or $\pm 10 \%$ of the corresponding estimates of Kemfert (1998). Results that satisfy these conditions are reported in boldface; results that are not in line with economic theory (such as negative elasticities of substitution) are reported in italics. 


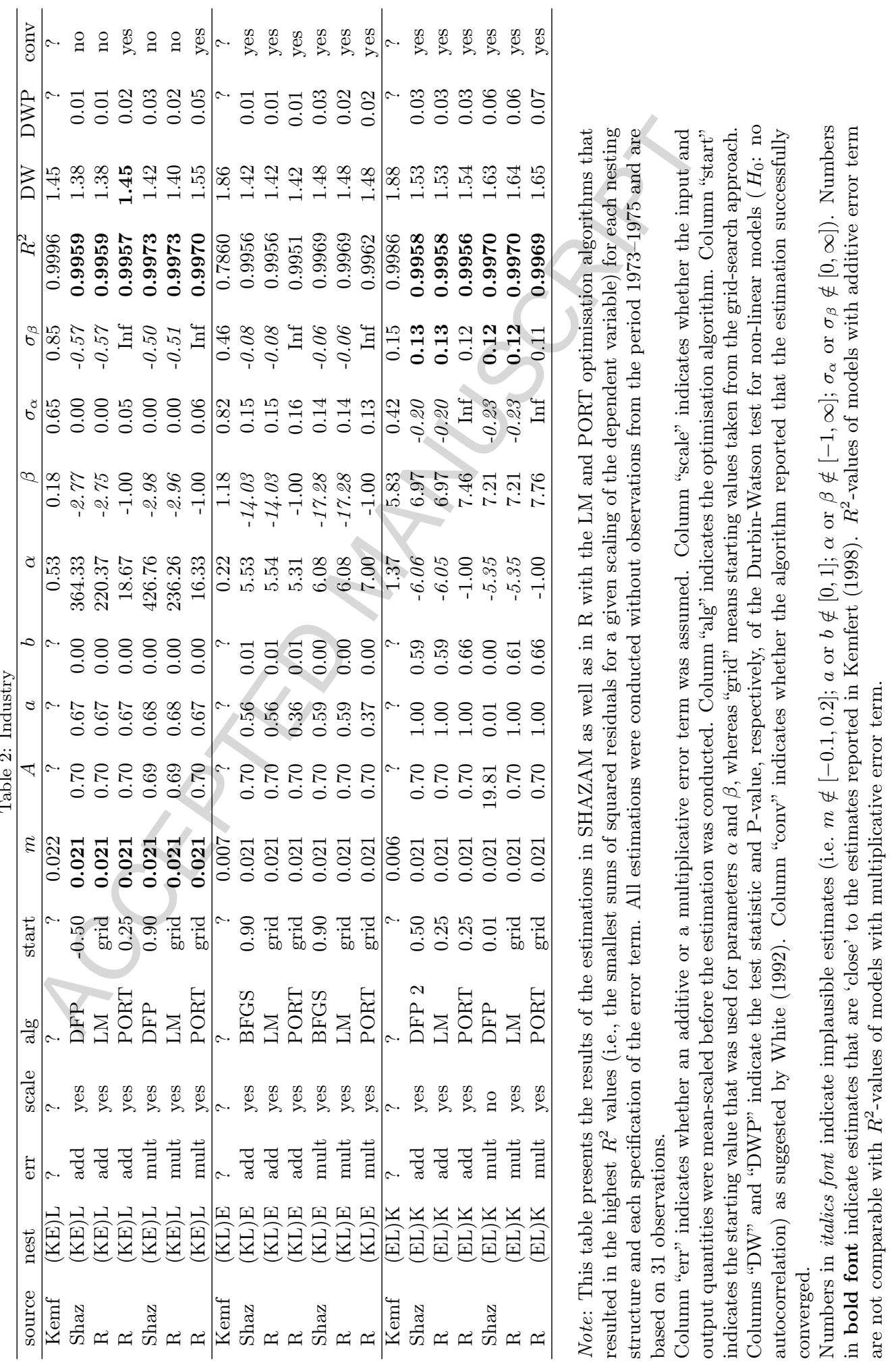


As Tables 2 and B.2 to B.8 show, only very few of our estimated parameters are close to those reported in Kemfert (1998) and we are unable to fully replicate any of the 24 estimations ( 8 sectors $\times 3$ nesting structures) that are reported in Kemfert (1998) - neither when using SHAZAM nor when using R. Tables B.9 to B.56 in the On-line Appendix indicate that even when using the same data set and the same software as in Kemfert (1998) and a vast amount of variations in starting values, optimisation algorithms, scaling of the variables, and specifications of the error term, none of our regressions more or less replicates any of the 24 estimation results reported in Kemfert (1998).

Furthermore, as Tables 2 and B.2 to B.8 show, not a single unrestricted estimation (i.e., using SHAZAM or $\mathrm{R}$ with the LM algorithm) returns a set of estimates that is consistent with economic theory, i.e., at least one share parameter is smaller than zero or larger than one or at least one elasticity of substitution is negative. Also, for most of the sub-sectors and nesting structures, the $R^{2}$ values of our estimated models reported in tables 2 and B.2 to B. 8 deviate markedly from those reported in Kemfert (1998), which clearly indicates that the optimisation algorithm used in that article reached a different optimum than ours. However, a remarkable finding is that even for estimation models that produce $R^{2}$-values close to the values reported in Kemfert (1998) - see for example the results for German Industry in Table 2- estimates of the substitution parameters and elasticities of substitution still deviate strongly from the results reported in Kemfert (1998).

While Kemfert (1998, p. 254) reports that there is "no indication of positive or negative first order autocorrelation of the residuals," we find significantly positive first-order autocorrelation of the residuals in almost all model specifications for the sector German Industry (see Table 2) and in some model specifications of the Food and Iron sub-sectors (see Tables B.4 and B.5 in the On-line Appendix, respectively). ${ }^{11}$

Although our estimates markedly deviate from the results in the original article, the estimates from the unrestricted estimation models in SHAZAM and R (with algorithm LM) are often identical or very close. The stability of our results indicates (i) that the starting values we have chosen in SHAZAM in many cases enable SHAZAM to find the global minimum of the objective function (as indicated by the grid search in $\mathrm{R}$ ) and

\footnotetext{
11 In addition to testing for autocorrelation in the residuals, we tested for (non-)stationarity of the data and the residuals. Several of the input and output quantities that are used in the estimations are non-stationary (see Tables E.1 and E.2 in the On-line Appendix). Standard procedures for dealing with non-stationary variables in econometric estimations, e.g., taking first differences, can only be applied to linear models and, thus, are not applicable to estimations of non-linear CES functions. However, in spite of the non-stationarity of several of the input and output quantities, the residuals of the estimated models are generally stationary (see Tables B.9 to B.56 in the On-line Appendix). Given that the residuals are autocorrelated but stationary, the standard errors of the estimated parameters are downward biased, i.e., the parameters estimates are less precise than indicated by their standard errors, but the estimates are still unbiased and consistent.
} 
(ii) that irrespective of which optimisation algorithm we use, there is little ambiguity regarding the parameter values at the global minimum.

Based on the available information in Kemfert (1998) we can merely hypothesise about the causes for these deviations. Three possible explanations seem most obvious, but are by no means meant to be exclusive.

One possible explanation for the deviations between our results and those reported in Kemfert (1998) could be that Kemfert (1998) may have used non-mean-scaled variables, which can result in suboptimal estimates due to numerical problems (see section 3.2). Indeed, as indicated by Tables B.9 to B.56, whilst regression results based on meanscaled data are very stable, some regressions of exactly the same model and data with non-mean-scaled variables result in a wide parameter range for the estimates.

Another possibility for the deviation in the estimation results could be that the results reported in Kemfert (1998) actually constitute a local minimum, i.e., that the algorithm only converged to a local minimum within proximity to the starting values. As the article does not report the starting values for the non-linear optimisation, nor how these starting values are derived, we have no possibility to test whether we can replicate the reported parameters in case they result from an eventual local minimum close to the starting values. However, this explanation is contradicted by the - for the most partlarger $R^{2}$ values reported in Kemfert (1998), which indicate estimates with a smaller sum of squared residuals than the estimates found in our regressions with SHAZAM and through the grid search in $\mathrm{R}$.

Finally, a third possible explanation for these findings could lie in methodological differences between Kemfert (1998) and our approach that have not been reported in the original article and, hence, have not been implemented in the replication.

To summarise: we are unable to replicate most of the results reported in Kemfert (1998), irrespective of whether we use the SHAZAM software with various algorithms, starting values, and error term specifications, or whether we use improved procedures in R. Whilst we are uncertain about the exact cause of the differences of our results, it is important to keep in mind that, as concluded in section 3.1, the data in Kemfert (1998) are compromised and will lead to unreliable estimates of the substitution parameters. In the next section, we present results from estimates using a new data set.

\section{Replication using current data}

In order to present updated elasticities of substitution, we created a new data set for the German Industry and key sub-sectors for years 1991-2014. In this section, we describe how we constructed this data set and present and discuss the updated elasticities of substitution. 


\subsection{Data}

In order to improve upon the results reported in Kemfert (1998), we tried to correct the issues in her data set that we discussed in section 3.1. Unfortunately, it proved impossible to find reliable data on energy expenditures for the period of 1960 to 1980 that we would need to correct the dependent variable. In order to still present an updated set of substitution elasticities, we collected a more recent data set for the German industry, as well as for eight major sub-sectors, for the years 1991 to 2014 . $^{12}$ We acquired data on gross value added (in bil. EUR and as deflated chain index), net capital stock (in bil. EUR and as deflated chain index), labour (in thsd. persons employed), and energy consumption (in TJ), as well as energy costs (in thsd. EUR) from the German Statistical Office (DESTATIS) both at the industrial level as well as the sectoral level (see Table A.3 in the On-line Appendix for a more detailed overview of the data and the data sources). The sectors included are: the chemical sector (including the pharmaceutical industry), the mining sector, the food sector (including the tobacco industry), the paper sector, the metal producing and processing sector, the vehicle producing sector, the machinery producing sector, and the electronics sector (including products for the production and transportation of electricity, e.g., transmission lines) - see Table A.4 of the On-line Appendix for a more detailed overview of the sub-sectors. These sub-sectors cover most of the manufacturing sector, as well as the mining sector, and stand for approximately $80 \%$ of the energy consumption of the entire industry.

We created the dependent variable for our analysis by adding real energy costs (at 2010 prices) to the real value added (at 2010 prices) and we used the deflated net capital, the work force (in thsd. persons employed) and energy consumption (in TJ) as inputs (see section A.3 of the On-line Appendix for more details on the construction of the data set). The resulting data clearly show a drop in value added and energy use for most sectors in the year 2009, resulting from the economic crisis. There appears only one outlier in the data (a 35\% jump in energy use in the Paper sector in 2005, followed by a $16 \%$ drop). The capital-value added ratios $(K / Y)$ range from 1.8 to 7.4 , which is in line with the data sets discussed in section 3.1 (except for the data in Kemfert, 1998).

\subsection{Results and discussion}

We estimate CES functions using aggregate data on the entire industrial sector, data for each of the eight sub-sectors, and a pooled data set of all eight sub-sectors. We only

12 The German Federal Statistical Office provides data on West Germany that we would need for a replication study only up to 1994, whilst it provides data for entire Germany (i.e., East and West Germany) only from 1991 onwards. Furthermore, there have been changes in both sector definitions and definitions of variables as well as data construction methods at the Statistical Office since the publication of Kemfert (1998). 
used mean-scaled data. All estimations were conducted in $\mathrm{R}$ using the same optimisation algorithms and approaches as outlined in section 3.2.3. We again estimated all three nesting structures with both an additive and a multiplicative specification of the error term.

We summarize the estimated elasticities of substitution in Table 3. Detailed estimation results are presented in Tables C.1 to C.10. When estimating the CES function without restrictions (i.e., using the LM algorithm), the majority of the estimated models is not in line with economic theory, i.e., at least one share parameter is smaller than zero or larger than one or at least one elasticity of substitution is negative. ${ }^{13}$ In those cases, where the parameters obtained in an unrestricted estimation are consistent with economic theory, the unrestricted estimates (i.e., using the LM algorithm) are mostly very close to the restricted estimates (i.e., using the PORT algorithm).

In Table 3, we present the elasticities of substitution that are based on the estimates obtained with the PORT algorithm, because they are consistent with economic theory. One observation from these Tables is that many estimated elasticities of substitution are extremely large or even infinite, which indicates (nearly) perfect substitutability between the inputs. This is particularly the case for the substitution between labour and energy in the (entire) industrial sector as well as in the Mining, Paper, and Vehicle sub-sectors. This is caused by substitution parameters, $\alpha$ and $\beta$, that are at the lower boundary of our parameter restrictions, i.e., very close or equal to -1 .

Another observation from Table 3 and Tables C.1 to C.10 in the On-line Appendix is that many standard errors of the estimated share parameters, substitution parameters, and elasticities of substitution are very large. ${ }^{14}$ This indicates that the data used for the estimations in many cases does not provide sufficient information to precisely identify these parameters and elasticities. This is likely caused by the rather small sample size (24 observations for the entire industrial sector and the eight sub-sectors, 192 observations for the pooled data set), the rather small (relative) variations in the explanatory variables, the very high correlation between some of the explanatory variables, ${ }^{15}$ as well as the nested CES functional form. For a given set of input quantities, this functional form often

\footnotetext{
13 A similar conclusion was drawn in Koesler and Schymura (2015) for regressions without parameter restrictions. Like us, they proceed to report results based on the PORT algorithm with parameter restrictions.

14 Durbin-Watson tests indicate that the residuals of several estimated models (particularly for the Chemical, Machines, Metal, and Vehicle sub-sectors and the pooled data set) are autocorrelated (see Tables C.1 to C.10). Given that autocorrelated residuals result in downward-biased standard errors, the parameter estimates of these models are even less precise than indicated by the reported standard errors.

15 For instance, the coefficient of correlation between the time trend and the capital input of the Vehicle sub-sector and the coefficient of correlation between the labour input and energy input of the mining sub-sector are both 0.98 (see Tables D.9 to D.16 for all coefficients of correlation between the explanatory variables).
} 
predicts a nearly constant output quantity for a wide range of parameter combinations. Hence, a wide range of parameter combinations gives nearly the same fit of the model and minor stochastic influences (e.g., truly random measurement errors in the observed output quantity which are absorbed in the error term) can have large effects on the combination of parameter values that gives the best fit of the model. In these situations, it is not unlikely that minor stochastic influences push parameters outside the economically meaningful ranges to give the best fit of the model, even though the (unknown) 'true' parameters are within economically meaningful ranges.

Table 4 compares our estimated elasticities of substitution for the pooled data set (which is based on the largest number of observations) with estimated elasticities from all other publications that we found that use a 'direct' approach to estimate a nested CES function with capital, labour, and energy for industrial sectors or entire economiesincluding Kemfert (1998). Although the estimation results come from different countries, it is surprising how much the value of each elasticity of substitution varies over these studies. For instance, for the (KL)E nesting structure, the elasticity of substitution between energy and the other inputs as found in other studies - which is very important in modelling the costs of climate policy - ranges from 0.0061 (i.e., nearly a Leontief functional form) for the UK to 1.75 (i.e., a much easier substitution than assumed in a Cobb-Douglas functional form) for Portugal. Although the firms in the UK and Portugal likely apply different technologies, it is questionable whether the substitutability between energy and other inputs differs so substantially between these two countries. Furthermore, although Kemfert (1998) and Kemfert and Welsch (2000) use the same approach, the same software, and data from the same country, some estimated elasticities of substitution differ markedly between these two studies, e.g., Kemfert (1998) estimates $\sigma_{(L E) K}=0.146$, whilst Kemfert and Welsch $(2000)$ estimates $\sigma_{(L E) K}=0.899$.

Although the elasticities of substitution estimated in other studies tend to have smaller standard errors than ours, many of these standard errors are still rather large. For instance, Kemfert and Welsch (2000) estimate $\sigma_{(K E) L}=0.529$ with an (approximate) standard error of 0.4172 , which implies a $95 \%$ confidence interval of $(-0.29,1.35)$. This includes both a Leontief functional form $\left(\sigma_{(K E) L}=0\right)$ and easier substitution than a Cobb-Douglas functional form $\left(\sigma_{(K E) L}>1\right)$. Note that Heun et al. (2017) do not report standard errors in their paper, while Koesler and Schymura (2015) separately estimate a CES function for value added and one that combines value added with materials and energy. 
Table 3: Estimated Elasticities of Substitution (new data set, PORT algorithm)

\begin{tabular}{|c|c|c|c|c|c|c|c|c|c|}
\hline sector & nest & err & & $\sigma_{E K}$ & & $\sigma_{E L}$ & & $\sigma_{K L}$ & $n$ \\
\hline Industry & $(\mathrm{KE}) \mathrm{L}$ & add & 1.11 & $(\infty)$ & $\infty$ & & $\infty$ & $(-)$ & 24 \\
\hline Industry & $(\mathrm{KE}) \mathrm{L}$ & mult & 0.07 & $(\infty)$ & $\infty$ & $(-)$ & $\infty$ & $(-)$ & 24 \\
\hline Industry & $(\mathrm{KL}) \mathrm{E}$ & add & $\infty$ & $(-)$ & $\infty$ & $(-)$ & 0.91 & $(\infty)$ & 24 \\
\hline Industry & $(\mathrm{KL}) \mathrm{E}$ & mult & $\infty$ & $(-)$ & & $(-)$ & 0.11 & $(\infty)$ & 24 \\
\hline Industry & $(\mathrm{EL}) \mathrm{K}$ & add & 1.11 & $(\infty)$ & & $(-)$ & 1.11 & $(\infty)$ & 24 \\
\hline Industry & $(\mathrm{EL}) \mathrm{K}$ & mult & 0.07 & $(\infty)$ & $\infty$ & $(-)$ & 0.07 & $(\infty)$ & 24 \\
\hline Chemical & $(\mathrm{KE}) \mathrm{L}$ & add & 0.04 & $(0.10)$ & 0.80 & $(\infty)$ & 0.80 & $(\infty)$ & 24 \\
\hline Chemical & $(\mathrm{KE}) \mathrm{L}$ & mult & 0.04 & $(0.15)$ & 0.13 & $(\infty)$ & 0.13 & $(\infty)$ & 24 \\
\hline Chemical & $(\mathrm{KL}) \mathrm{E}$ & add & 0.04 & $(0.15)$ & 0.04 & $(0.15)$ & 0.80 & $(\infty)$ & 24 \\
\hline Chemical & $(\mathrm{KL}) \mathrm{E}$ & mult & 0.04 & $(0.21)$ & 0.04 & $(0.21)$ & 0.13 & $(\infty)$ & 24 \\
\hline Chemical & $(\mathrm{EL}) \mathrm{K}$ & add & 0.04 & $(0.09)$ & 0.38 & $(\infty)$ & 0.04 & $(0.09)$ & 24 \\
\hline Chemical & $(\mathrm{EL}) \mathrm{K}$ & mult & 0.04 & $(0.15)$ & 0.19 & $(\infty)$ & 0.04 & $(0.15)$ & 24 \\
\hline Electric & $(\mathrm{KE}) \mathrm{L}$ & add & $\infty$ & $(-)$ & 0.07 & $(0.19)$ & 0.07 & $(0.19)$ & 24 \\
\hline Electric & $(\mathrm{KE}) \mathrm{L}$ & mult & 0.02 & $(0.74)$ & 0.24 & $(0.36)$ & 0.24 & $(0.36)$ & 24 \\
\hline Electric & $(\mathrm{KL}) \mathrm{E}$ & add & 0.43 & (1.01) & 0.43 & (1.01) & $\infty$ & $(-)$ & 24 \\
\hline Electric & $(\mathrm{KL}) \mathrm{E}$ & mult & 0.26 & $(0.42)$ & 0.26 & $(0.42)$ & 0.01 & $(279.00)$ & 24 \\
\hline Electric & $(\mathrm{EL}) \mathrm{K}$ & add & $\infty$ & $(-)$ & 0.20 & $(0.53)$ & $\infty$ & $(-)$ & 24 \\
\hline Electric & $(\mathrm{EL}) \mathrm{K}$ & mult & 0.02 & (1.45) & 0.26 & $(0.42)$ & 0.02 & (1.45) & 24 \\
\hline Food & $(\mathrm{KE}) \mathrm{L}$ & add & 0.04 & $(0.06)$ & 0.03 & $(0.23)$ & 0.03 & $(0.23)$ & 24 \\
\hline Food & $(\mathrm{KE}) \mathrm{L}$ & mult & 0.04 & $(0.06)$ & 0.02 & $(0.26)$ & 0.02 & $(0.26)$ & 24 \\
\hline Food & $(\mathrm{KL}) \mathrm{E}$ & add & $\infty$ & $(-)$ & $\infty$ & $(-)$ & 0.00 & $(0.39)$ & 24 \\
\hline Food & $(\mathrm{KL}) \mathrm{E}$ & mult & 0.67 & (13.85) & 0.67 & (13.85) & 0.00 & $(0.34)$ & 24 \\
\hline Food & (EL)K & add & 0.04 & $(0.05)$ & $\infty$ & $(-)$ & 0.04 & $(0.05)$ & 24 \\
\hline Food & (EL)K & mult & 0.04 & $(0.05)$ & $\infty$ & $(-)$ & 0.04 & $(0.05)$ & 24 \\
\hline Machines & $(\mathrm{KE}) \mathrm{L}$ & add & 0.11 & $(0.30)$ & 1.25 & $(\infty)$ & 1.25 & $(\infty)$ & 24 \\
\hline Machines & $(\mathrm{KE}) \mathrm{L}$ & mult & 0.11 & $(0.28)$ & 1.25 & $(\infty)$ & 1.25 & $(\infty)$ & 24 \\
\hline Machines & $(\mathrm{KL}) \mathrm{E}$ & add & 0.11 & $(0.53)$ & 0.11 & $(0.53)$ & 0.79 & $(\infty)$ & 24 \\
\hline Machines & $(\mathrm{KL}) \mathrm{E}$ & mult & 0.11 & $(0.22)$ & 0.11 & $(0.22)$ & 0.07 & $(\infty)$ & 24 \\
\hline Machines & $(\mathrm{EL}) \mathrm{K}$ & add & 0.11 & $(0.22)$ & 0.07 & $(\infty)$ & 0.11 & $(0.22)$ & 24 \\
\hline Machines & $(\mathrm{EL}) \mathrm{K}$ & mult & 0.11 & $(0.24)$ & 2.00 & $(\infty)$ & 0.11 & $(0.24)$ & 24 \\
\hline Metal & $(\mathrm{KE}) \mathrm{L}$ & add & 0.07 & $(\infty)$ & 0.04 & $(0.03)$ & 0.04 & $(0.03)$ & 24 \\
\hline Metal & $(\mathrm{KE}) \mathrm{L}$ & mult & 0.83 & $(\infty)$ & 0.04 & $(0.03)$ & 0.04 & $(0.03)$ & 24 \\
\hline Metal & $(\mathrm{KL}) \mathrm{E}$ & add & 0.04 & $(0.03)$ & 0.04 & $(0.03)$ & 0.79 & $(\infty)$ & 24 \\
\hline Metal & $(\mathrm{KL}) \mathrm{E}$ & mult & 0.04 & $(0.03)$ & 0.04 & $(0.03)$ & 0.21 & $(\infty)$ & 24 \\
\hline Metal & $(\mathrm{EL}) \mathrm{K}$ & add & 0.77 & $(\infty)$ & 0.04 & $(0.03)$ & 0.77 & $(\infty)$ & 24 \\
\hline Metal & $(\mathrm{EL}) \mathrm{K}$ & mult & 0.63 & $(\infty)$ & 0.04 & $(0.03)$ & 0.63 & $(\infty)$ & 24 \\
\hline Mining & $(\mathrm{KE}) \mathrm{L}$ & add & 0.10 & $(0.19)$ & 0.00 & $(0.11)$ & 0.00 & $(0.11)$ & 24 \\
\hline
\end{tabular}




\begin{tabular}{|c|c|c|c|c|c|c|c|c|c|}
\hline Mining & $(\mathrm{KE}) \mathrm{L}$ & mult & 0.00 & $(0.31)$ & $\infty$ & $(-)$ & $\infty$ & $(-)$ & 24 \\
\hline Mining & $(\mathrm{KL}) \mathrm{E}$ & add & $\infty$ & $(-)$ & $\infty$ & $(-$ & 0.01 & $(3.28)$ & 24 \\
\hline Mining & $(\mathrm{KL}) \mathrm{E}$ & mult & $\infty$ & $(-)$ & $\infty$ & ( & 0.03 & $(0.14)$ & 24 \\
\hline Mining & $(\mathrm{EL}) \mathrm{K}$ & add & 0.04 & $(0.07)$ & $\infty$ & & 0.04 & $(0.07)$ & 24 \\
\hline Mining & $(\mathrm{EL}) \mathrm{K}$ & mult & 0.03 & $(0.12)$ & $\infty$ & $(-$ & 0.03 & $(0.12)$ & 24 \\
\hline Paper & $(\mathrm{KE}) \mathrm{L}$ & add & 0.83 & $(\infty)$ & $\infty$ & $(-)$ & $\infty$ & $(-)$ & 24 \\
\hline Paper & $(\mathrm{KE}) \mathrm{L}$ & mult & 0.09 & $(\infty)$ & $\infty$ & ( & $\infty$ & $(-)$ & 24 \\
\hline Paper & $(\mathrm{KL}) \mathrm{E}$ & add & $\infty$ & $(-)$ & $m$ & 1 & 0.11 & $(\infty)$ & 24 \\
\hline Paper & $(\mathrm{KL}) \mathrm{E}$ & mult & $\infty$ & $(-)$ & & $(-)$ & $\infty$ & $(-)$ & 24 \\
\hline Paper & $(\mathrm{EL}) \mathrm{K}$ & add & 0.83 & $(\infty)$ & 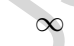 & $(-)$ & 0.83 & $(\infty)$ & 24 \\
\hline Paper & $(\mathrm{EL}) \mathrm{K}$ & mult & 0.08 & $(\infty)$ & $\infty$ & $(-)$ & 0.08 & $(\infty)$ & 24 \\
\hline Vehicles & $(\mathrm{KE}) \mathrm{L}$ & add & 1.67 & $(\infty)$ & $\infty$ & $(-)$ & $\infty$ & $(-)$ & 24 \\
\hline Vehicles & $(\mathrm{KE}) \mathrm{L}$ & mult & 29.10 & & $\infty$ & $(-)$ & $\infty$ & $(-)$ & 24 \\
\hline Vehicles & $(\mathrm{KL}) \mathrm{E}$ & add & $\infty$ & 1 & $\infty$ & $(-)$ & 0.08 & $(\infty)$ & 24 \\
\hline Vehicles & $(\mathrm{KL}) \mathrm{E}$ & mult & $\infty$ & ( & $\infty$ & $(-)$ & 0.33 & $(\infty)$ & 24 \\
\hline Vehicles & $(\mathrm{EL}) \mathrm{K}$ & add & 0.87 & $(\infty)$ & $\infty$ & $(-)$ & 0.87 & $(\infty)$ & 24 \\
\hline Vehicles & $(\mathrm{EL}) \mathrm{K}$ & mult & 0.31 & $(\infty)$ & $\infty$ & $(-)$ & 0.31 & $(\infty)$ & 24 \\
\hline Pooled & $(\mathrm{KE}) \mathrm{L}$ & add & 0.06 & $(0.12)$ & 1.00 & $(0.53)$ & 1.00 & $(0.53)$ & 192 \\
\hline Pooled & $(\mathrm{KE}) \mathrm{L}$ & mult & & $(-)$ & $\infty$ & $(-)$ & $\infty$ & $(-)$ & 192 \\
\hline Pooled & $(\mathrm{KL}) \mathrm{E}$ & add & 0.06 & $(0.13)$ & 0.06 & $(0.13)$ & 2.00 & $(1.53)$ & 192 \\
\hline Pooled & $(\mathrm{KL}) \mathrm{E}$ & mul & $\infty$ & $(-)$ & $\infty$ & $(-)$ & $\infty$ & $(-)$ & 192 \\
\hline Pooled & $(\mathrm{EL}) \mathrm{K}$ & add & 1.42 & $(1.63)$ & $\infty$ & $(-)$ & 1.42 & $(1.63)$ & 192 \\
\hline Pooled & $(\mathrm{EL}) \mathrm{K}$ & mult & 1.31 & $(1.15)$ & $\infty$ & $(-)$ & 1.31 & $(1.15)$ & 192 \\
\hline
\end{tabular}

Note: Years included in estimation: 1991-2014. If the standard errors are not presented (-), they could not be calculated due to (near) singularity of the Hessian matrix. Values larger (smaller) than (minus) one thousand are abbreviated by " $\infty$ " ("- $\infty$ "). 


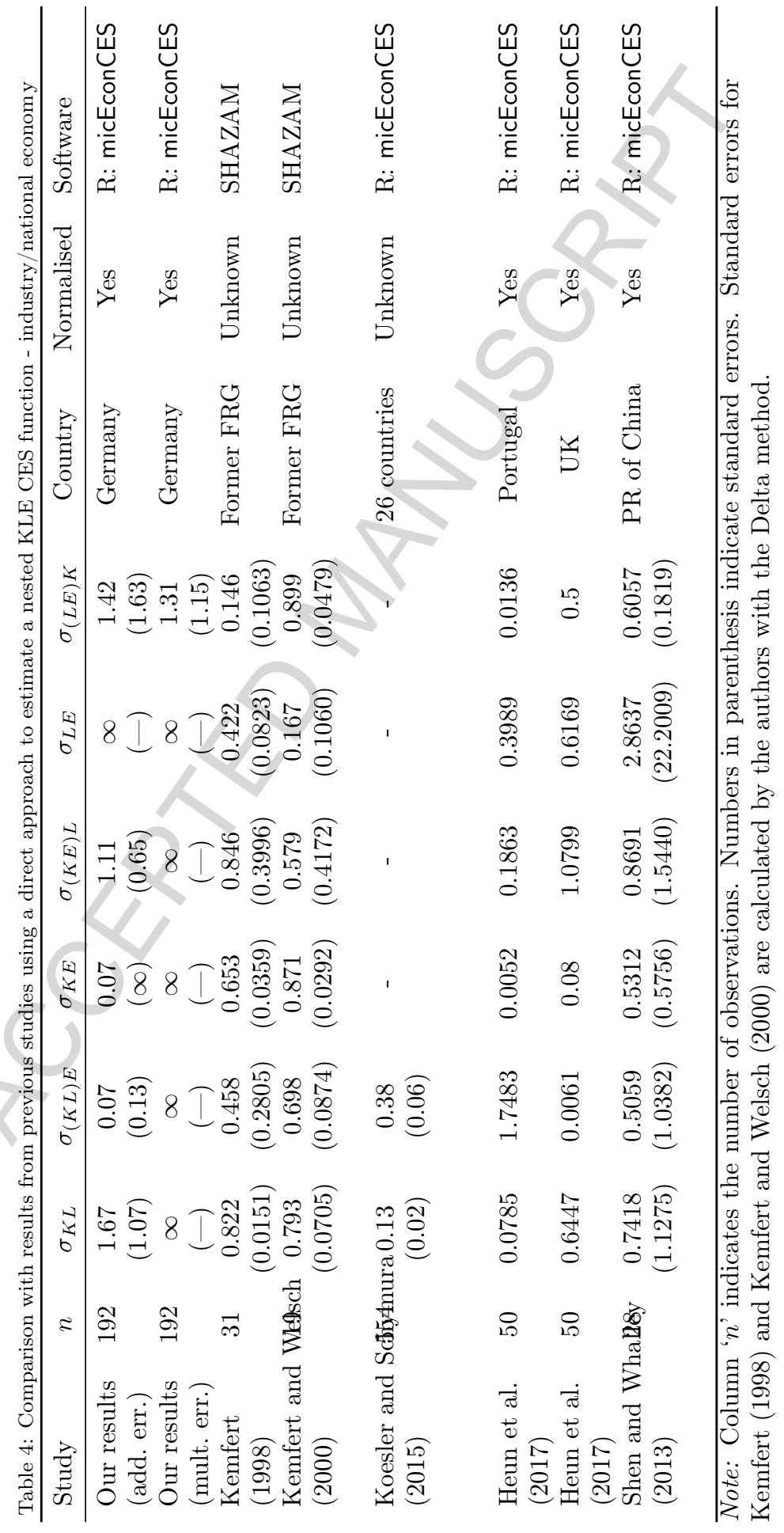




\section{Conclusions}

The results of climate policy simulation models can be rather sensitive to the values of the elasticities of substitution between capital, labour and energy (Jacoby et al., 2006). Hence, such models require reliable estimates for these elasticities.

In this paper we have shown that the results reported in Kemfert (1998) cannot be considered to be reliable due to a range of problems with the data used. Furthermore, we have tried to replicate the results reported in that paper using two different statistical software packages and various combinations of assumptions regarding the error term, starting values, and scaling of the data. Although some of our estimated parameters are close to the results in Kemfert (1998), this is not the case for most parameters. Hence, in general we were unable to replicate the results reported in Kemfert (1998). Furthermore, when estimating models without parameter restrictions, the regression results are all inconsistent with economic theory.

In order to present updated elasticities, we created a new data set covering the years 1991-2014 and important industrial sectors in Germany. We find that many standard errors are very large, which indicates that the data used often does not provide sufficient information to precisely identify the substitution and share parameters.

We conclude that none of our estimated elasticities - neither based on the data in Kemfert (1998), nor based on our newly created dataset-are reliable and can be used in policy modeling. Our findings, and those of previous studies following the same approach, are sobering and indicate severe inherent problems with estimating nested CES production functions of the KLE type through a direct approach, in particular when using short time series (as, e.g., in Kemfert, 1998; Shen and Whalley, 2013, or in this study). As regressions of nested CES functional forms have objective functions with vast flat surfaces in combination with severely cratered surfaces, they require (i) large data sets and (ii) large independent variations in the explanatory variables (i.e., without too much multicollinearity) in order to obtain reliable estimates. Short time series neither provide a sufficiently large number of observations nor sufficient independent variation of the explanatory variables over time to fulfill this requirement. Therefore, we argue that a large part of the variation in the estimates from different studies is simply driven by stochastic disturbances.

Given our and other authors' findings, we carefully advise against the use of a 'direct' approach for estimating capital-labour-energy substitution in a nested CES functional form, unless these data requirements are met (as maybe in Koesler and Schymura, 2015, as they use pooled data). For shorter time series the 'economic approach' as discussed in Van der Werf (2008) — although having its own shortcomings - may for the time being be the better choice to estimate a three-input nested CES function. Alternatively, 
Bayesian econometric approaches with informative priors based on existing information on the production technology and/or economic behaviour could help to obtain more reliable estimates, e.g., by circumventing problems of estimates out of boundaries or at the boundaries of the economically meaningful parameter ranges.

Given the importance of valid estimates for substitution elasticities in climate and energy policy simulations, future research should investigate the advantages and disadvantages of various approaches for estimating nested CES functions.

Acknowledgements and declaration of interest: The authors thank the German Federal Statistical Office (DESTATIS) for their valuable help during the data collection and Christian Elleby and two anonymous reviewers for their very helpful comments and suggestions. Of course all errors remain the sole responsibility of the authors.

The authors declare no conflict of interest whilst conducting this study.

\section{References}

Bauer, N., Baumstark, L., Leimbach, M., 2012. The REMIND-R model: the role of renewables in the low-carbon transformation - first-best vs. second-best worlds. Climatic Change 114, 145-168.

Brockway, P. E., Heun, M. K., Santos, J., Barrett, J. R., 2017. Energy-extended CES aggregate production: Current aspects of their specification and econometric estimation. Energies 10, 1-23.

Carrara, S., Marangoni, G., May 2017. Including system integration of variable renewable energies in a constant elasticity of substitution framework: The case of the WITCH model. Energy Economics 64, $612-626$.

Chang, K.-P., 1994. Capital-energy substitution and the multi-level CES production function. Energy Economics $16(1), 22-26$.

Dong, Y., Whalley, J., November 2011. Carbon motivated regional trade arrangements: Analytics and simulations. Economic Modelling 28, 2783-2792.

Gay, D. M., 1990. Usage summary for selected optimization routines. Tech. Rep. 153, AT\&T Bell Laboratories.

Henningsen, A., Henningsen, G., 2011a. Econometric estimation of the constant elasticity of substitution function in R: Package micEconCES. Tech. rep., FOI Working Paper 2011/9, Institute of Food and Resource Economics, University of Copenhagen.

Henningsen, A., Henningsen, G., 2011b. micEconCES: analysis with the constant elasticity of substitution CES function. Tech. rep., R Package Version 0.9.

Henningsen, A., Henningsen, G., 2012. On estimation of the CES production function - Revisited. Economics Letters 115, 67-69.

Heun, M. K., Santos, J., Brockway, P. E., Pruim, R., Domingos, T., Sakai, M., 2017. From theory to econometrics to energy policy: Cautionary tales for policymaking using aggregate production functions. Energies 10, 1-44.

Jacoby, H. D., Reilly, J. M., McFarland, J. R., Paltsev, S., 2006. Technology and technical change in the MIT EPPA model. Energy Economics 28, 610-631.

Judge, G. G., Hill, R. C., Griffiths, W., Lütkepohl, H., Lee, T.-C., 1982. Introduction to the Theory and Practice of Econometrics. John Wiley and Sons, New York. 
Kelley, C. T., 1999. Iterative Methods of Optimization. SIAM Society for Industrial and Applied Mathematics, Philadelphia.

Kemfert, C., 1998. Estimated substitution elasticities of a nested CES production function approach for Germany. Energy Economics 20, 249-264.

Kemfert, C., Welsch, H., 2000. Energy-capital-labor substitution and the economic effects of $\mathrm{CO}_{2}$ abatement: Evidence for Germany. Journal of Policy Modeling 22 (6), 641-660.

Klump, R., de La Grandville, O., 2000. Economic growth and the elasticity of substitution: Two theorems and some suggestions. American Economic Review 90 (1), 282-291.

Klump, R., McAdam, P., Willman, A., 2011. The normalized CES production function theory and empirics. Tech. Rep. 1294, European Central Bank Working Paper Series.

Koesler, S., Schymura, M., 2015. Substitution elasticities in a constant elasticity of substitution framework - empirical estimates using nonlinear least squares. Economic Systems Research 27 (1), $101-121$.

Marquardt, D. W., 1963. An algorithm for least-squares estimation of non-linear parameters. Journal of the Society for Industrial and Applied Mathematics 19, 431-441.

Meng, S., 2014. How may a carbon tax transform Australian electricity industry? A CGE analysis. Applied Economics 46, 796-812.

Prywes, M., 1986. A nested CES approach to capital-energy substitution. Energy Economics 8, 22-28.

R Core Team, 2017. R: A Language and Environment for Statistical Computing. R Foundation for Statistical Computing, Vienna, Austria.

URL http: //www.R-project.org/

Shen, K., Whalley, J., 2013. Capital-labor-energy substitution in nested CES production functions for China. Tech. Rep. 19104, NBER Working Paper Series.

Timmer, M., Erumban, A., Reitze, G., Los, B., Temurshoev, U., de Vries, G., Arto, I., Andreoni, V., Genty, A., Neuwahl, F., Rueda-Cantuche, J., Villanueva, A., 2012. The World Input-Output Database (WIOD): Contents, sources and methods, Groningen, the Netherlands, University of Groningen.

Turner, K., Hanley, N., 2011. Energy efficiency, rebound effects and the environmental Kuznets Curve. Energy Economics 33, 709-720.

Van der Werf, E., November 2008. Production functions for climate policy modeling: an empirical analysis. Energy Economics 30 (6), 2964-2979.

White, K. J., 1992. The durbin-watson test for autocorrelation in nonlinear models. The Review of Economics and Statistics 74 (2), 370-373. URL http: //www.jstor.org/stable/2109675

Wissema, W., Dellink, R., 2007. AGE analysis of the impact of a carbon energy tax on the Irish economy. Ecological Economics 61 (4), 671-683. 